\title{
Timing properties of gamma-ray bursts detected by SPI-ACS detector onboard INTEGRAL
}

\author{
V. Savchenko ${ }^{1,2}$, A. Neronov ${ }^{1,2}$, and T. J.-L. Courvoisier ${ }^{1,2}$ \\ 1 ISDC Data Centre for Astrophysics, Ch. d'Ecogia 16, 1290 Versoix, Switzerland \\ 2 Observatory of Geneve, University of Geneva, Ch. des Maillettes 51, 1290 Sauverny, Switzerland \\ e-mail: volodymyr. savchenko@unige.ch
}

Received 24 January 2012 / Accepted 5 March 2012

\begin{abstract}
Aims. We study timing properties of a large sample of gamma-ray bursts (GRB) detected by the anti-coincidence shield (ACS) of the SPI spectrometer of INTEGRAL telescope.

Methods. We identify GRB-like events in the SPI-ACS data. The data set under investigation is the history of count rate of the SPI-ACS detector recorded with a binning of $50 \mathrm{~ms}$ over the time span of $\sim 10 \mathrm{yr}$. In spite of the fact that SPI-ACS does not have imaging capability, it provides high statistics signal for each GRB event, because of its large effective area.

Results. We classify all isolated excesses in the SPI-ACS count rate into three types: short spikes produced by cosmic rays, GRBs and Solar flare induced events. We find some $\sim 1500$ GRB-like events in the $10 \mathrm{yr}$ exposure. A significant fraction of the GRB-like events identified in SPI-ACS occur in coincidence with triggers of other gamma-ray telescopes and could be considered as confirmed GRBs. We study the distribution of durations of the GRBs detected by SPI-ACS and find that the peak of the distribution of long GRBs is at $\simeq 20$ s, i.e. somewhat shorter than for the long GRBs detected by BATSE. Contrary to the BATSE observation, the population of short GRBs does not have any characteristic timescale. Instead, the distribution of durations extends as a powerlaw to the shortest timescale accessible for SPI-ACS, $\leq 50 \mathrm{~ms}$. We also find that a large fraction of long GRBs has a characteristic variability timescale of the order of $1 \mathrm{~s}$. We discuss the possible origin of this timescale.
\end{abstract}

Key words. gamma rays: general - gamma-ray burst: general

\section{Introduction}

Gamma-ray bursts (GRBs) are the most violent explosions in the universe. They are among the first cosmic $\gamma$-ray sources discovered more than 40 years ago (Klebesadel et al. 1973). In spite of the long observation history, the nature of the GRB phenomenon remains obscure. A number of missions were dedicated to the study of the physical properties of GRBs. The isotropy of GRB distribution, indicating their extragalactic origin, was established by CGRO/BATSE observations (Paciesas et al. 1999). Long-lasting afterglows discovered by BeppoSAX (Piro et al. 1998) confirmed the cosmological nature of GRB phenomenon and provided an insight in the energy budget of GRBs comparable to the energy release in the core collapse of massive stars. Complex time structure of GRB lightcurves, with variability down to millisecond timescale was revealed by CGRO/BATSE (Bhat et al. 1992). Existence of an additional delayed emission component in the $\mathrm{GeV}$ energy band was discovered by CGRO/EGRET (Schneid et al. 1992) and was recently confirmed by Fermi/LAT (Abdo et al. 2009).

The transient nature of GRBs makes the information available about each individual event usually very fragmentary: a complete picture of the GRB phenomenon could not be established based on the observations of individual events. Instead, the complete picture could be obtained via population study in which different events appear as manifestations of a single GRB phenomenon. The population study should in the first place clarify if all the observed GRBs are produced by one and the same physical phenomenon or if the set of GRBs consists of several sub-populations, which might possibly have different physical origin. This problem is not simple: the quality of the data on more numerous weak bursts is not sufficient for quantitative analysis, while the bright bursts are not frequent enough to form a good statistical sample.

Up to now, the largest sample of GRBs available for a statistical study has been collected by the CGRO/BATSE (Paciesas et al. 1999) and Konus-Wind (Aptekar et al. 1995). One of the main findings of BATSE and Konus-Wind was the discovery of a dichotomy in the distribution of GRB durations (Mazets et al. 1981; Kouveliotou et al. 1993). This distribution reveals two broad bumps. The first bump is at the durations $T_{90}<2 \mathrm{~s}$ (where $T_{90}$ is the time over which $90 \%$ of the GRB fluence is registered), corresponding to the "short" GRBs. The second bump is at $T_{90}>2 \mathrm{~s}$, corresponding to the "long" GRBs. In the BATSE GRB sample, the population of long GRBs peaked at $T_{90} \simeq 50 \mathrm{~s}$, while the typical timescale of the short GRBs was $T_{90} \simeq 0.3 \mathrm{~s}$ (Kouveliotou et al. 1993). Further analysis of a larger sample of BATSE bursts using improved duration estimates found that the durations of the long bursts seem to peak in 30-40 s range rather then at $50 \mathrm{~s}$ (Paciesas et al. 1999).

The division of GRB in two sub-populations is supported by observation of differences in spectral properties of the two sub-classes: the short GRBs have harder spectra (Kouveliotou et al. 1993). Long GRBs are characterized by larger spectral lags (Norris et al. 2001). The spatial locations of the bursts from the two sub-classes in their host galaxies are also different: long GRBs are tracing the star formation while the short GRBs are not correlated with the star formation regions (Barthelmy et al. 2005). 
The short versus long dichotomy is commonly believed to be related to the difference in burst progenitors. The long GRBs seem to be associated with the final stage of evolution of massive stars: gravitational collapse of the stellar core and/or supernova explosion (MacFadyen \& Woosley 1999). The short GRBs are possibly related to coalescence of compact objects - neutron stars or black holes (Barthelmy et al. 2005). In a number of cases supernovae associated with long GRBs (Woosley \& Bloom 2006) were directly observed. Direct confirmation of the hypothesis of association of the short GRBs to the merger events is still missing.

Some problems of the division of the entire GRB populaiton in two classes remain (Zhang 2011). In particular, tight upper limits on the optical flux of several long GRBs exclude supernova as the GRB progenitor (Fynbo et al. 2006; Gal-Yam et al. 2006; Valle et al. 2006). A peculiar long GRB with firmly excluded supernova progenitor, GRB 060614A (Gehrels et al. 2006) has also negligible spectral lag typical for the short bursts. It is also possible that a separate population of softer spectra GRB-like events exists, the X-ray flashes (XRF). It is, however, not clear if the XRFs are just a softer tail of the GRB distribution (Heise et al. 2001) or whether they are a different phenomenon (Soderberg et al. 2006). Large fraction of short GRBs may contain softer extended emission component, making their total duration as large as tens of seconds (Norris \& Bonnell 2006). In such situation the possibility of division of the GRB population in "short" and "long" sub-classes becomes problematic. As claimed by Cline et al. (2007), the shortest bursts, with durations of less than $100 \mathrm{~ms}$, may also posses remarkably distinct properties: unusual spectra and non-isotropic angular distribution (other studies of anisotropy of GRB subpopulations did not, however, come to the same conclusion, Briggs et al. 1996), a luminosity distribution suggesting Galactic origin. A population of Galactic sources, soft gamma-repeaters (SGR), is known to produce short GRB-like events (Hurley et al. 2005, 2009; Savchenko et al. 2010). The brightest of these events could be detected by existing instruments even if they occur outside the Milky Way, in the nearby galaxies. This type of events could constitute a non-negligible fraction of the observed short GRB sample (Hurley et al. 2005, 2009).

The focus of the present study is on the timing properties of GRBs detected by the anti-coincidence shield (ACS) of spectrometer SPI onboard the INTEGRAL satellite (von Kienlin et al. 2003). Although SPI-ACS does not have an imaging capability, it has a large effective area (up to about $1 \mathrm{~m}^{2}$ ). SPI-ACS readout does not rely on any onboard trigger criteria and instead continuously measures the event rate all over the INTEGRAL mission lifetime with a time binning of $50 \mathrm{~ms}$.

The first attempt to systematically study GRBs with SPIACS was done by Rau et al. (2005) using about 800 days of data. This study considered 179 GRBs also detected by other satellites, but visible in the SPI-ACS. Unlike Rau et al. (2005), we primarly rely on identification of the GRBs based solely on the ACS data, so that our GRB sample is not dependent on the data of other satellites. This allows us to perform a study of the timing properties of GRBs, which is not affected by the selection biases (sensitivity, trigger configurations) of other telescopes. We separately discuss a sub-sample of GRBs confirmed by the other satellites (Konus/Wind, Fermi/GBM, RHESSI, Suzaku/WAM, Swift/BAT, INTEGRAL/ISGRI, AGILE, IPN network).

In the following sections we describe in detail the algorithm of detection of bursts in the SPI-ACS data and the classification of events into three classes: GRBs, instrumental short spikes and Solar flares. For each GRB we measure its duration and investigate if the GRB lightcurve possesses a characteristic timescale other than the overall duration. Based on this study we build the distribution of GRB durations and characteristic timescales.

The main obstacle for the study of GRBs with SPI-ACS is the presence of the so-called "spikes", which have ligthcurves similar to the short GRBs, but are, most probably, associated to cosmic rays hitting the ACS detector (Rau et al. 2005). Association of the spikes with the cosmic ray events was questioned by Deluit et al. (2004), who argued that the cosmic ray interactions in the SPI-ACS could not manifest themselves as events with high count statistics. Instead, Deluit et al. (2004) have advanced a hypothesis of possible existence of real very short GRBs missed by the other instruments. Later, Minaev et al. (2010) stacked the profiles of a sample of unidentified short bursts in the SPI-ACS and found a weak but recognizable afterglow of the duration of more than a second. They interpreted this observation as an evidence in favor of the hypothesis of the association of the (fraction of) spikes with the real short GRBs.

Our study of the origin of the short spikes reported below, agrees with the principal conclusion of Rau et al. (2005) that the spikes are events induced by high-energy cosmic rays. At the same time, we show that the instrument effects of the ACS, the decay of cosmic ray induced radioactivity in BGO crystals, produces the "afterglow" of the spikes discussed by Minaev et al. (2010). The detailed knowledge of properties of the spikes allows us to fully characterize them and to separate them from the real GRBs in our analysis.

\section{The INTEGRAL/SPI-ACS}

The spectrometer SPI (Vedrenne et al. 2003) onboard INTEGRAL Winkler et al. (2003) is surrounded by an active ACS (von Kienlin et al. 2003), consisting of 91 (only 89 currently functional) $\mathrm{BGO}$ (Bismuth Germanate, $\mathrm{Bi}_{4} \mathrm{Ge}_{3} \mathrm{O}_{12}$ ) scintillator crystals. The SPI-ACS also serves as a large effective area (up to $\sim 1 \mathrm{~m}^{2}$ ) $\gamma$-ray detector with a quasi-omnidirectional field of view (von Kienlin et al. 2003). The design of the ACS is such that it has (almost) no sensitivity to the direction of $\gamma$-rays.

The exact magnitude of the SPI-ACS effective area and its dependency on the direction and the energy is not well-known. It can be investigated through detailed simulations of the photon propagation in the detector, as it was done, for example by Mereghetti et al. (2009). However, this requires a mass model of the entire INTEGRAL satellite. An alternative method consists in making use of the events detected simultaneously by SPI-ACS and other detectors. This approach was exploited by Viganò \& Mereghetti (2009).

The ACS data are event rates integrated over all the scintillator crystals with a time resolution of $50 \mathrm{~ms}$. The typical number of counts per $50 \mathrm{~ms}$ time ranges from about 3000 to 6000 (or even more during high Solar activity).

The unique property of the SPI-ACS data is that, contrary to other existing GRB detectors, the readout does not rely on any trigger, so that a complete history of the detector count rate over the $10 \mathrm{yr}$ period is recorded. This opens the possibility of an offline search of GRBs in the compete data sample.

The main challenge for the detection of GRBs in the countrate data of SPI-ACS is in the separation of real GRBs from other sources of variability of the signal, like the high-energy cosmic ray hits or charged particle events from the Solar flares. In the imaging detectors of GRBs, such separation could be done based on the directionality of $\gamma$-rays contributing to the event. In 
Table 1. Comparison of SPI-ACS to other GRB detectors.

\begin{tabular}{|c|c|c|c|c|c|}
\hline & "Suzaku/HXD-II WAM & CGRO/BATSE LAD & ВерpоSAX/PDS GRBM & "INTEGRAL/SPI-ACS & Fermi/GBM \\
\hline Crystal & BGO & $\mathrm{NaI}(\mathrm{T} 1)$ & CsI(Na) & BGO & BGO \\
\hline Energy range (keV) & $50-5000$ & $20-2000$ & $40-700$ & $>75$ & $150-30000$ \\
\hline Effective area $\left(\mathrm{cm}^{2}\right)$ & 800@100 keV & 2000@100 keV & 700@200 keV & 3000@100keV & $120 @ 200 \mathrm{keV}$ \\
\hline & 400@1 MeV & 150@1 MeV & 100@1 MeV & \$8000@1MeV & $120 @ 1 \mathrm{MeV}$ \\
\hline Time resolution & $31.25 \mathrm{~ms}$ & $2 \mathrm{~ms}$ & $7.8 \mathrm{~ms}$ & $50 \mathrm{~ms}$ & $5 \mu \mathrm{s}$ \\
\hline
\end{tabular}

the SPI-ACS separation of GRBs has to be done based on different selection criteria.

The burst like events are detected on top of a steady background. The nearly constant background evolves only by a factor of two over the ten years of operation. The background rate increases during the periods of passage of INTEGRAL through the Earth radiation belt and during the SPI "annealing" phases. These periods are excluded from the analysis reported below.

The SPI-ACS data are searched for bursts in real time as part of the telescope operations, using a dedicated INTEGRAL Burst Alert System (IBAS) (Mereghetti et al. 2003). The SPI-ACS triggers are used in the Inter-Planetary Network (IPN; Hurley et al. 2004) to derive the burst positions by the triangulation if other satellites of the IPN also detect the burst.

Comparison of SPI-ACS to other similar scintillator type GRB detectors is given in Table 1. From this table one could see that the only GRB detector with the effective area comparable to that of the SPI-ACS was BATSE onboard the CGRO mission.

\section{Detection of GRBs}

We use the SPI-ACS data of 1030 revolutions of the INTEGRAL satellites, from 20 to 1100 (3230 days from 2002-12-12 to 201110-16). Some revolutions are excluded entirely because of the high solar activity or SPI annealing. The remaining total exposure time is 2412 days.

\subsection{Detection algorithm}

To identify burst-like events on top to the average background of SPI-ACS, we build the running mean of the ACS count rate on 15 different timescales, from $100 \mathrm{~ms}$ to $10^{4} \mathrm{~s}$. At each timescale $\delta t_{\mathrm{s}}$, the estimate of background count rate is obtained from the current value of the count rate on larger timescales, $\delta t_{\mathrm{b}}>\delta t_{\mathrm{s}}$. The burst-like events in the lightcurve with the time binning $\delta t_{\mathrm{s}}$ are identified as excesses in the count rate over the background count rate estimated in this way. Significance of detection of the burst detection depends, in general on the timescale $\delta t_{\mathrm{b}}$ used for the background rate estimate. We consider a burstlike event appearing on a timescale $\delta t_{\mathrm{s}}<20 \mathrm{~s}$ as significantly detected if there is at least one scale $\delta t_{\mathrm{b}}$ for which the significance of the signal detection on top of the estimated background exceeds a pre-defined threshold. The pair $\delta t_{\mathrm{s}}, \delta t_{\mathrm{b}}$ for which the significance of detection of the burst is the highest defines the "best timescales" and this highest detection significance is considered as the burst detection significance.

This burst detection method includes an optimization for the signal/background timescales combination. This introduces a trial factor, which has to be taken into account in the significance estimate. Taking this into account, we set the detection significance threshold at a relatively high value, $6 \sigma$, which corresponds to $\approx 5 \sigma$ after the account of the trial factor.

The brightest bursts affect the running-mean estimates of the background even on the largest timescales. To correct for this effect, we perform the detection procedure twice, excluding the bursts detected at the first stage from the background estimation at the second stage.

The background count rate is additionally affected by Solar activity. We evaluate the Solar activity related variability of the background via monitoring of the excess variance of the background count rate on the "best" background timescale $\delta t_{\mathrm{b}}$ for every burst. The significance of the burst detection is corrected taking into account possible excess variance of the background.

\subsection{Duration measurements}

The most common and straightforward definition of the burst duration is the length of the time interval in which the burst signal is detected as an excess over the background. With this definition, the measurement of the burst duration is obtained via a measurement of the significance of the burst signal in each bin of the lightcurve. The duration of the burst depends on the detection significance threshold adopted in the analysis. This means that the burst duration defined in this way is not necessarily the characteristics of the burst itself, but depends on the sensitivity of the instrument. Another drawback of this definition of the burst duration is that it could not take into account correlations between adjacent time bins. This leads to systematic underestimation of the duration: the burst could be not detectable in the narrow time bins, but still be significantly detectable on several time bins scale.

An alternative technique used to estimate burst durations is Bayesian block analysis (Scargle 1998). It consists in approximating the burst lightcurve with a sequence of intervals with constant flux. At first one interval is assumed. In each step of the iterative procedure one boundary is introduced. The iterations stop, when no more divisions are supported by the likelihood ratio test. This method has the advantage of not relying on an a-priori known background. This is particularly useful when the background is rapidly changing like in BAT and GBM. One of the disadvantages of the method is that it adopts a model of the signal in each block (constant), which is not physically motivated. This could lead to an underestimation of the duration in the case of long and weak bursts.

The method in which the burst duration is calculated based on significance of detection in fixed time bins could be improved to take into account weaker signals in several adjacent time bins. For this we run the analysis on several different predefined timescales. The set of the timescales is the same as used in the detection, see Sect. 3.1. We calculate the error on the burst durations from the comparison of the durations obtained assuming different detection thresholds (4, 3 and 2 sigma). In general, durations calculated using different binning of the lightcurve are different. We compare the durations calculated for different binnings and choose the timescale, which provides the minimal uncertainty to measure the start and stop of the burst.

Similarly to the Bayesian blocks methodology, the methods adopting binning of the lightcurves implicitly assume constant 
rate within the bin. Physically motivated models of the bursts profiles can be more successful in interpreting the properties of the burst prompt emission lightcurve. For example, profiles of the burst may be fitted with a collection of pulses (Norris et al. 1996). However, more complex models the compromise generality of the analysis.

To verify the precision of the measurement of the burst durations, we perform an analysis based on the fixed time bins and on the Bayesian blocks and compare the results. We find that the burst durations found using the two methods are consistent within the uncertainties.

The duration methods discussed above are based on statistical testing of the signal over the background. Since different instruments have different sensitivity to the signal and different background levels, the durations measured in this way depend on the instrument performance. A definition of the burst duration, which is less dependent on the instrument sensitivity is the so-called $T_{90}$-duration, which is equal to the time during which $90 \%$ of the burst flux arrives. To measure $T_{90}$ we identified the total flux within the detected duration, and adjust the start and the stop until 5\% of the flux is removed from the beginning and from the end of the burst.

\subsection{Types of burst-like events in SPI-ACS}

The total number of burst-like events found in our analysis is 82231. Most of these events are a special type of events called "short spikes" (Rau et al. 2005). At long timescales, variations of the SPI-ACS count rate are mostly due to the changes of Solar activity. Below we explain the algorithm used to separate the GRBs from the spikes at short timescales and from the Solar flares at long timescales.

\subsubsection{Solar activity}

The Solar activity in the SPI-ACS introduces variability of various magnitudes, ranging from the minor flickering and slow evolution to giant flares increasing the SPI-ACS background by factor of few for days. As mentioned above, the variability of the background due to the solar activity poses a problem for the correct calculation of significance of the detection of GRBs. Our procedure for the calculation of significance of the burst takes into account the excess variance of the background during the periods of Solar activity.

Lightcurves of some solar flares could sometimes appear similar to those of GRBs. It is not possible to distinguish such flares from GRBs based on the SPI-ACS data alone. To distinguish the Solar flares from the GRBs we use the data of monitoring of the Solar activity by the solar weather prediciton center (SWPC) ${ }^{1}$ to exclude time intervals, which might be affected by the Solar activity.

\subsection{Short spikes}

The majority of burst-like events in SPI-ACS are "short spikes", with typical durations of about the about $50-100 \mathrm{~ms}$, i.e. one or two bins of the lightcurve. The rate of these events is very high about 30 spikes/day (Rau et al. 2005; Deluit et al. 2004). Spikes are usually detected only in one $50 \mathrm{~ms}$ bin and very infrequently in two, which suggests that the real duration is much smaller than the bin size.

\footnotetext{
${ }^{1}$ http://www.swpc.noaa.gov/
}

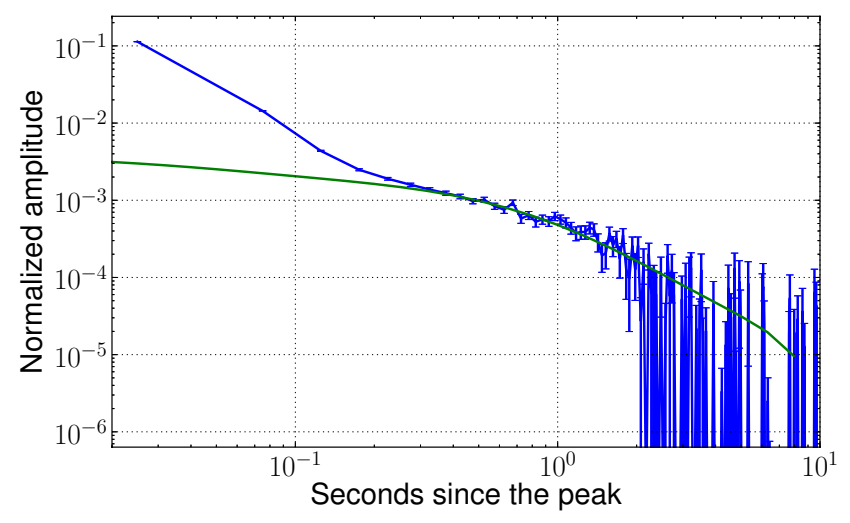

Fig. 1. The stacked profile of the spikes (blue data points). The extended tail is fitted with the decay of residual cosmic ray induced radioactivity of the BGO found from FLUKA simulations (green curve).

The nature of these events was controversial. The analysis by Rau et al. (2005) suggests that the spikes are produced following hits of the ACS by high-energy cosmic rays. The observed flux of high-energy cosmic rays is (Adriani et al. 2011)

$F_{\mathrm{CR}} \simeq 10^{2}\left[\frac{E_{\mathrm{CR}}}{10 \mathrm{TeV}}\right]^{-1.7} \mathrm{~m}^{-2} \mathrm{sr}^{-1} \mathrm{~d}^{-1}$.

Taking into account the effective area of ACS, the observed rate of spikes suggests that the events are produced by cosmic rays with energies above $\sim 30 \mathrm{TeV}$.

Alternatively, the spikes (or a fraction of them) could be real GRBs, which escape detection by other instruments. This could arise because of large effective area of ACS at the energies above $\sim 1 \mathrm{MeV}$. If the spikes are real GRBs, they should have very hard intrinsic spectrum, to avoid detection by other instruments. The hard and very short bursts would be a phenomenon of particular interest. A distinct population of bursts with similar properties might have been found in the Swift/BAT, BATSE and Konus Wind burst samples (Cline et al. 1999, 2007).

We dedicate the next Section to study of the Short Spike properties.

\section{The nature of the short spikes}

The properties of the short spikes reveal a remarkable stability over the whole mission span (10 years of data). While the background is slowly changing with the solar cycle, the sensitivitycorrected spike rate remains constant. This observation makes a Solar origin of spikes unlikely, unless there is a high-energy solar phenomenum not modulated with solar cycles. If cosmic rays are resposible for the spikes, however, they should not be affected by the solar activity and thus have energy higher than $\gtrsim 10 \mathrm{GeV}$, where the Solar modulation effect vanishes (Parker 1958; Shikaze et al. 2007).

To characterize the time profile of the spikes we stack the lightcurves of all the very short bursts detected by SPI-ACS (shorted then $0.5 \mathrm{~s}$ ) shifted in time to match the bin with the highest countrate.

The stacking analysis reveals a clearly recognizable weak $(0.1 \%$ of the total number of counts or just a few counts in a single spike on average) afterglow lasting for almost $3 \mathrm{~s}$ (Fig. 1). From this figure one could see that the "prompt" phase of the spike is characterized by an exponential rise/exponential decay profile. The rise and decay time are constrained to be shorter than $\sim 25 \mathrm{~ms}$, half of the time bin of the ACS lightcurve. The 
overall event number and count statistics in the stacked sample is largely dominated by spikes, so that the presence of confirmed short GRBs or SGR flares in the sample does not affect the average spike profile found from the stacking analysis. To verify that the long tail of the stacked profile is not due to the presence of a sub-population of rare real GRBs in the stacked sample, we studied distribution of countrates in individual events in the time bins of the tail. This distribution follows a Gaussian with the center displaced from zero. If the tail of the profile would be due to the presence of a small number of real GRBs, the distribution would be instead a Gaussian centered at zero, with a small excess at positive count rates. We have also verified that the profiles of individual brightest spikes follow closely the averaged stacked profile, with a clearly visible tail.

Rau et al. (2005) suggested that the spikes are produced following energy deposition in the ACS by a high-energy cosmic ray. The cosmic ray initiates a shower of secondary particles through pair production and bremmstralung and then propagating further to the Ge detectors of SPI and saturating them. One of the arguments presented by Rau et al. (2005) in favor of interpretating of the spikes as events produced by the high-energy cosmic rays is the correlation of the spikes with events of saturation of the SPI detector. Rau et al. (2005) noticed that saturation of at least one Ge detectors of the SPI often (but not always) accompanies a short spike and that saturation of more than one SPI detector is always accompanied by a spike-like increase in SPI-ACS count-rate.

Our analysis of the correlation between the spikes and the saturation of SPI detectors does not fully confirm the observation of Rau et al. (2005). Although we find a significant correlation between the spikes and SPI saturation moments (1600 coincidence events with expected 40), we find that saturations of more than one SPI detector are not always accompanied by spikes.

In particular, the saturation of all the SPI detectors is always accompanied by a gap in the SPI-ACS data as well, so that no spikes could be recorded. Although there is a significant correlation between spikes and "cosmic-ray track-like" saturation events in SPI (more than 3 detectors in a row are saturated), some $60 \%$ of the track-like events are not accompanied by spikes. The correlation is still weaker in the saturation events, which do not have track-like appearance. Only $5 \%$ of those events correlate with the spikes.

Problems of this hypothesis were highlighted by Deluit et al. (2004). Deluit et al. (2004) have noticed that all the particles from a cosmic ray induced shower in ACS would be counted as just one particle, because of the finite time resolution of the ACS counting electronics $(0.6 \mu \mathrm{s})$. Indeed, the shower particles travel with a speed close to the speed of light. The transit time of the ACS is much shorter than the time resolution of the detector. This means that the cosmic ray induced shower could not produce a spike in the ACS count rate. The shower particles also could not be responsible for the second-long afterglow shown in Fig. 1, as highlighted by Minaev et al. (2010).

A potential way out of this problem is that the cosmic ray induced shower in ACS is able to excite long-lived phosphorescence of the detector. In this case the phosphorescence signal persists on longer timescale and could be time-resolved by the ACS front-end electronics. This effect is actually observed in another detector onboard INTEGRAL, PICsIT, in which strong $\left(\sim 2500\right.$ counts $\left.\mathrm{s}^{-1}\right)$ and short $(\$ 170 \mathrm{~ms})$ spikes are associated with cosmic ray induced showers (Segreto et al. 2003). Timetagging of photons and energy resolution of PICSIT allow identification of the spikes as the phosphorescence of the detectors following the cosmic ray hits.
SPI-ACS uses undoped BGO scintillators, featuring very rapid deexcitation - with a characteristic times of about 60-300 ns followed by weak, $0.005 \%$, residual phosphorescence on the timescale of $3 \mathrm{~ms}$. If the energy deposit by a cosmic ray is large enough, even weak residual phosphorescence signal could be above the trigger threshold of the ACS front-end electronics. In this case, the detector will continue to count events on the timescale up to the $\sim \mathrm{ms}$ following the CR hit. This would explain the appearance of a spike in the ACS count rate as suggested by Rau et al. (2005).

However, prolonged phosphorescence of BGO crystal could not be responsible for the spike afterglows shown in Fig. 1, because persistence of the phosphorescence signal on the $\sim$ second timescale would produce a flat constantly high countrate $R \sim$ $50 \mathrm{~ms} / 0.6 \mu \mathrm{s} \simeq 80000$ counts/bin on this timescale, rather than "spike-with-a-tail" lightcurve. Instead, the tail could be attributed to another feature of the BGO crystal, the induced radioactivity. Activation of the $\mathrm{BGO}$ crystals with the cosmic ray protons is known to contribute to the measured background (Truscott \& Dyer 1997). We have investigated the radioactivity induced in the BGO crystals by high-energy cosmic ray passage using FLUKA Monte-Carlo code (Battistoni et al. 2007; Ferrari et al. 2005). These simulations reveal that the main radioactive decay channel is the decay of ${ }^{8} \mathrm{Li}$ with the half-life time $838 \mathrm{~ms}$. There is also a number of other isotopes with comparable decay times although lower abundances. A fit of the observed time profiles of the extended tails of the spikes with the time profiles of decay of the cosmic ray induced radioactivity found from FLUKA simulations is shown in Fig. 1. One could see that the time profile of the tail is well explained by the exponential decay of the induced radioactivity in the BGO crystal.

To summarize, the observed properties of the spikes are consistent with the assumption that they are produced by highenergy cosmic rays passing through the ACS and inducing phosphorescence and radioactivity in the BGO crystals.

Knowledge of the physical origin of the spikes enables us to efficiently reject them in the analysis of the GRB sample. All the spikes are expected to have identical time profiles, with the overall normalization determined by the energy of the primary cosmic ray. Rejecting all the events, which have the time profiles consistent with that of the template spike profile shown in Fig. 1, will remove most of the spikes, while suppressing only a small fraction of the real GRBs, which occasionally have time profiles similar to the spikes (a pronounced peak followed by a tail with fixed fractional amplitude and decay time). Taking this into account, we compute the likelihood for each event to be a spike rather than real GRB. We calibrate this likelihood with simulations and choose the threshold to reject the spikes in the real data.

\section{Properties of GRBs detected by SPI-ACS}

\subsection{GRB durations}

Rejection of spikes and Solar flare events from the overall sample of burst-like events in SPI-ACS leaves a sample of 1416 GRB lightcurves available for the timing analysis. Figure 2 shows the distribution of durations $T_{90}$ for these GRBs. This distribution is compared to the distribution of durations of the spikes shown by the blue histogram in Fig. 3 .

One could see that the spikes dominate the total number of events with durations up to several seconds. However, the characteristic time profile of the spikes allows their efficient rejection even on the shortest timescales. In particular, a significant 


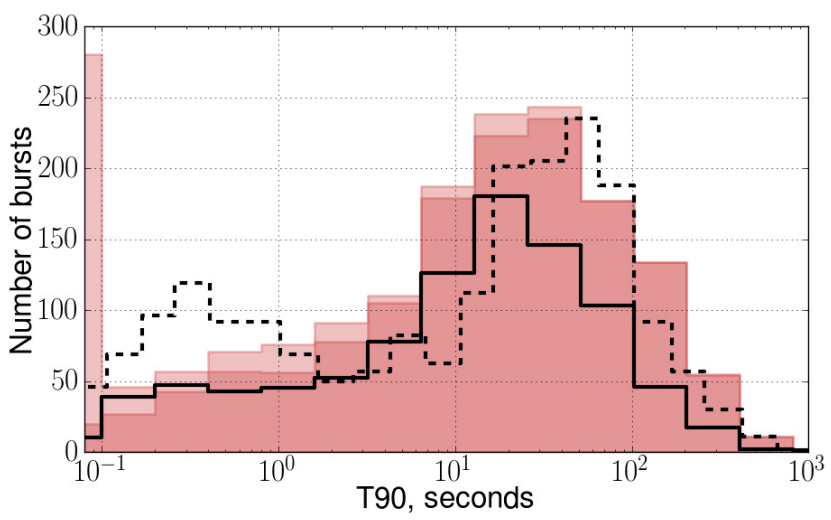

Fig. 2. Distributions of the $T_{90}$ durations. Dark and light shadings of the filled region correspond to 5 and 3 sigma confidence level for the event not being a spike. Solid black curve is for the confirmed bursts. The distribution of durations of GRBs from BATSE 4B GRB sample, scaled to match the normalization of the ACS sample, is shown by the black dashed line.

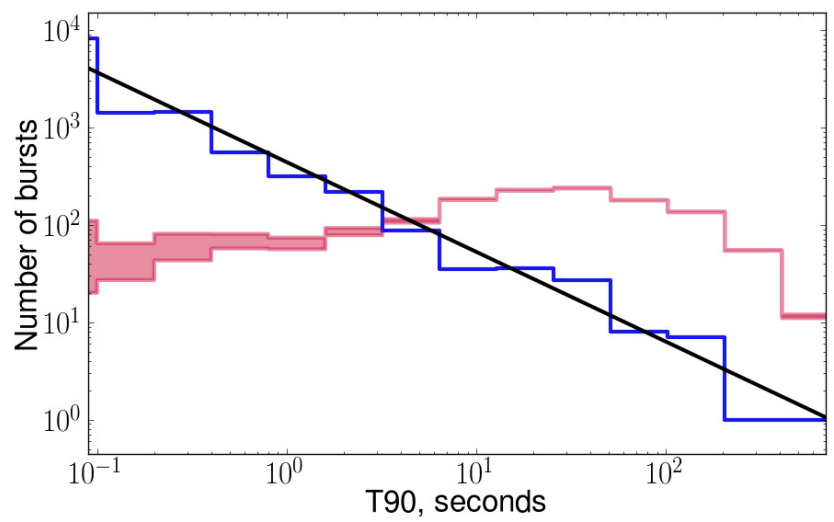

Fig. 3. Distribution of GRB durations (shaded region) compared to the distribution of durations of spikes (blue histogram).

number of GRBs with durations down to $\sim 100-150 \mathrm{~ms}$ is detected in the SPI-ACS data. Such bursts are identified as events for which the lightcurve is not consistent with the spike time profile. Two shaded histograms in Fig. 2 show two different confidence levels for an events not to be spikes. One could see that reducing the acceptance threshold leads to a sharp increase of the number of GRB-like events on the shortest timescale where difference between the spikes and real GRBs is more difficult to characterize. Taking this into account, we restrict our attention to GRBs with durations longer than $100 \mathrm{~ms}$ in the following.

Distribution of durations of the spikes follows a powerlaw on large timescales. This is explained by the fact that the span of the time interval during which the cosmic ray induced radioactivity produces a detectable signal in the BGO crystal scales proportionally to the overall number of the radioactive atoms, which is in turn proportional to the energy of the primary cosmic ray. Taking into account that the spectrum of cosmic rays follows a powerlaw, one could find that the distribution of durations of the spikes should also follow a powerlaw. Amplitude of statistical fluctuations of the spike duration distribution determines an upper bound on the possible number of real GRBs in each duration time bin. This upper limit is shown in Fig. 3 as an upper boundary of the pink-shaded region. Lower boundary of the pink shaded region is the distribution of the GRB-like events calculated with tight rejection threshold for the spikes, shown in Fig. 2.

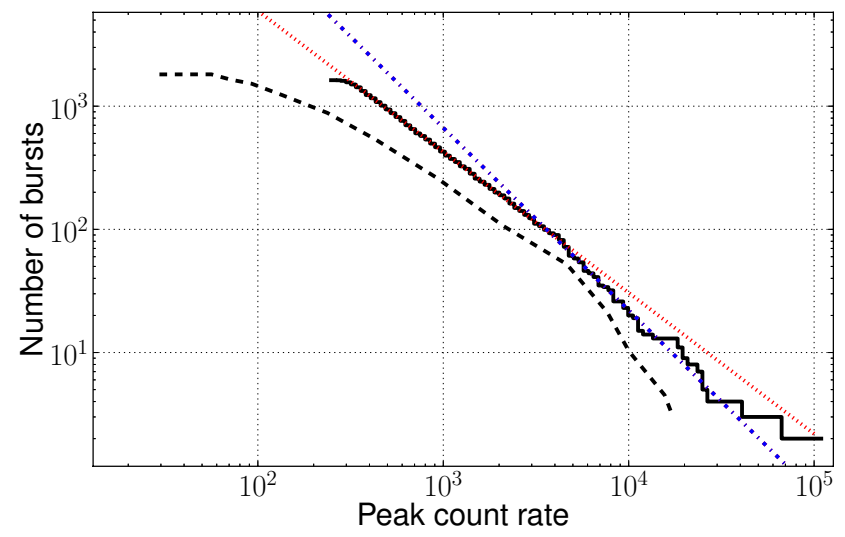

Fig. 4. Peak count rate distribution of the GRB candidate events. Powerlaw fits above and below the break at 4000 counts/s are shown with dotted lines. Distribution of the GRB peak fluxes as measured by BATSE (rescaled) is shown by the black dashed line.

Real GRBs are expected to produce signals not only in ACS, but also simultaneously in other GRB detectors. To verify the quality of our procedure of selection of GRB events from the set of burst-like events in SPI-ACS, we have searched for the events occurring in coincidence with triggers of other GRB detectors. Table 2 summarizes the result of our search. The number of events found in coincidence with triggers of other detectors is comparable to the number of events selected based on the SPIACS data alone. Slightly larger number of events found using SPI-ACS alone is readily explained by the large effetive area of ACS. In our search of events occurring in coincidence with triggers of other detectors, we have adjusted the search time window (within which the events are declared to be coincident) in such a way that the we expect 1 random coincidence.

The black solid histogram in Fig. 2 shows the distribution of durations of events occurring in coincidence with the triggers from other detectors. One could see that this distribution is consistent with that found based on the analysis of the SPI-ACS data alone, although with somewhat lower statistics.

Although the presence of the short spikes reduces the sensitivity of the SPI-ACS to the shortest $(<100 \mathrm{~ms})$ bursts, the number of short bursts with longer durations (100 ms-2 s) is rather well constrained. That distribution of durations of the independently detected bursts is so similar to the distribution of the confirmed burst durations (Fig. 2), shows that our spike filtering technique efficiently rejects most of the spikes and retains most of the bursts with durations longer than two ACS time bins.

\subsection{GRB peak fluxes}

To verify the efficiency of rejection of spikes based on the template profile, we study the distribution of peak countrates in the remaining GRB sample. The peak countrate measured by SPI-ACS provides an estimate of the peak flux of the GRB. SPI-ACS detector could not measure the peak flux of each detected GRB, because its effective area depends on the (in general, unknown) arrival direction of GRB photons. However, GRBs arrive isotropically, so that the average peak flux distribution could be measured for all the GRB population, using a known angle-averaged effecitve area of ACS. This distirbutiton is shown in Fig. 4. One could see that the distribution has the form of a broken powerlaw with slopes $-1.46 \pm 0.04$ at high countrates and $-1.12 \pm 0.01$ at the countrates below 4000 . This count rate corresponds to a GRB peak flux $\sim 10 \mathrm{ph} / \mathrm{cm}^{2} \mathrm{~s}$ in 
Table 2. SPI-ACS triggers detected simultaneously by other means.

\begin{tabular}{cccccccc}
\hline \hline & Konus/Wind & Suzaku/WAM & Fermi/GBM & RHESSI & Swift/BAT & IPN & Fermi/LAT \\
\hline GRB & 461 & 452 & 292 & 145 & 127 & 21 & 16 \\
\hline
\end{tabular}

the energy band above $80 \mathrm{keV}$ or, equivalently, to an energy flux $5 \times 10^{-6} \mathrm{erg} / \mathrm{cm}^{2} \mathrm{~s}$. For comparison, the distribution of the GRB peak fluxes as measured by BATSE (Kommers et al. 1998) is shown at the same figure. It is scaled for the convenience of the presentation. Notably, both the break and the slopes in the BATSE peak flux distribution are close to the one we observe.

\subsection{Characteristic timescale of long GRBs}

We have investigated the variability patterns of the GRB lightcurves, using conventional time series analysis tools, such as power spectral density (PSD) and structure funciton analysis. These tools are well suited for the analysis of long GRBs, in which the dynamic range of timescales/frequencies for each GRB event is large enough.

We use the first order structure function to quantify the burst temporal properties. The structure function analysis is analogous to the autocorrelation function (ACF) analysis, successfully used by a number of authors in investigations of the GRB variability (Borgonovo 2004; in 't Zand \& Fenimore 1996). We prefer the structure function analysis to the PSD analysis, which is better suited for the study of variability of persistent, rather than transient sources, because of the nature of the Fourier transform.

The structure function analysis could be used to identify a characteristic variability timescale, which appears as a localized "deep" feature superimposed on the powerlaw type behavior. We use this property of the structure function to investigate the possibile existence of a characteristic variability timescale of GRBs. The existence of such a timescale in the $T_{\text {var }} \simeq 1 \mathrm{~s}$ range was claimed by Beloborodov et al. (2000) based on the PSD analysis of a set of BATSE GRBs.

Our structurre function analysis confirms the result of Beloborodov et al. (2000). Figure 5 shows the characteristic timescales of long GRBs found form the structure function analysis and plotted as a function of the GRB duration. One could see that large fraction of GRBs has the characteristic timescale $T_{\text {var }}=1 \pm 1 \mathrm{~s}$.

\section{Discussion}

Our analysis of the sample of GRBs detected by SPI-ACS shows that the distribution of GRB durations shown in Fig. 2 has two components. Similarly to BATSE duration distribution, it has broad bump corresponding to the long GRB population. The typical duration of long GRBs in SPI-ACS $(\simeq 20 \mathrm{~s})$ is somewhat shorter than in BATSE $(\simeq 50 \mathrm{~s})$. This is in agreement with the previous studies of the SPI-ACS bursts performed by Rau et al. (2005). The difference in the typical duration could be attributed to the difference in the energy ranges in which BATSE and SPI-ACS are sensitive. The dependence of the duration distribution on the energy range was previously observed in Swift/BAT, BATSE, BeppoSAX and HETE data (Fenimore et al. 1995; Sakamoto et al. 2011). Longer bursts are known to have softer spectra. Besides, the spectra of individual GRBs have hard-tosoft evolution so that the burst duration grows with decreasing photon energy.

Contrary to BATSE burst duration distribution, the distribution of durations of SPI-ACS bursts, shown in Figs. 2 and 3,
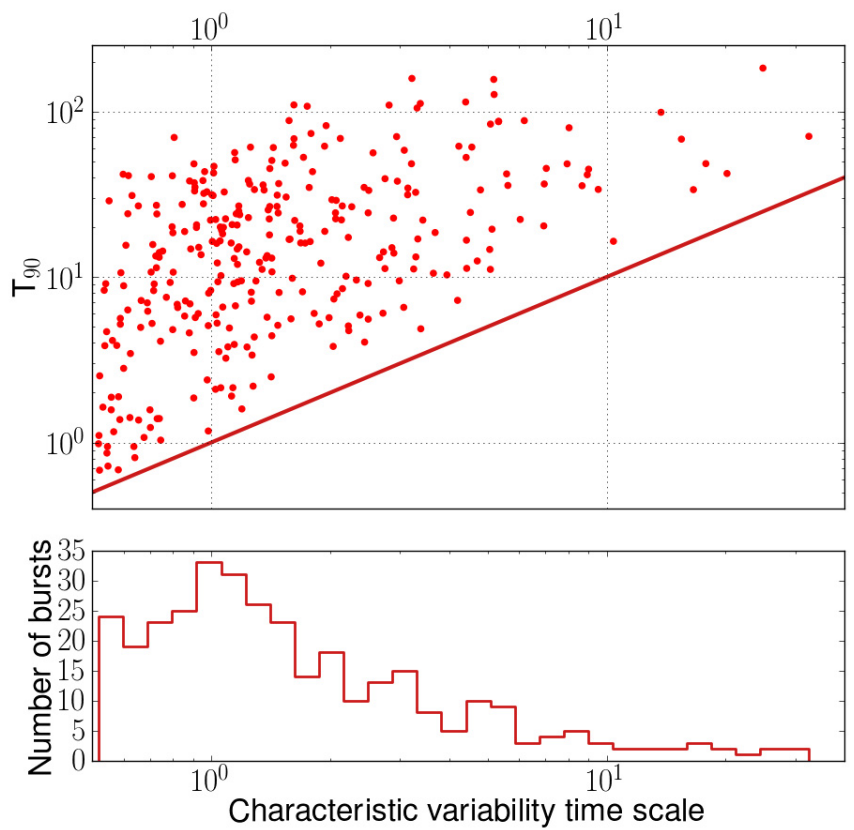

Fig. 5. Top panel: the first characteristic timescale vs. duration $\left(T_{90}\right)$ for long GRBs. Bottom panel: the distribution of the first characteristic timescales.

does not reveal short bursts concentrated around a characteristic timescale. Instead, the burst duration distribution follows a powerlaw at short timescales $T_{90} \lessgtr 3 \mathrm{~s}$. The powerlaw extends to the shortest timescale accessible to the ACS $(\simeq 100 \mathrm{~ms})$ and, most probably, to the longer timescales, beyond $\sim 3 \mathrm{~s}$.

This might be either due to the instrumental effects or might reflect the GRB physics. An instrumental effect could arise e.g. because of the difference of sensitivity between BATSE and SPI/ACS. If short GRBs typically have extended tails, which were not detected by BATSE, but are visible to SPI/ACS, part of the bursts, which might have been responsible to the short burst duration distribution bump at $0.3 \mathrm{~s}$, might appear in SPI/ACS as longer bursts in the $\sim 3 \mathrm{~s}$ duration range. Otherwise, the difference between the short and the long bursts may not be fully characterized by the criterion $T_{90} \lessgtr 2 \mathrm{~s}$, as suggested by BATSE distribution. Separation in the hardness and spectral lags have to be used to establish the separation between the two main varieties of the GRBs. As discussed by Zhang (2011), it may be natural to adopt a different classification scheme: to Type I ("compact star GRBs") and Type II ("massive star GRBs") bursts.

Although our analysis does not reveal a separate population of very short bursts with the durations $T_{90}<100 \mathrm{~ms}$, discussed by Cline et al. (2007), we could not completely rule out the existence of such sub-population. Such very short bursts could be easily confused with the cosmic ray induced spikes in ACS. To distinguish real bursts from the cosmic-ray induced spikes a more advanced analysis based on the simultanenous detections by the other intruments, e.g. Suzaku/WAM, has to be done.

The sample of the SPI-ACS bursts presented in this work is, of course, subject to observational biases related to the detection efficiency of our method. It was shown that in the case of BATSE specific detection efficiency issues have significant effect on the 
burst duration distribution, increasing the fraction of short bursts to about $40 \%$ (Lee \& Petrosian 1996). Unfortunately, low number of measurable burst characteristics accessible in SPI-ACS (limited to timing properties of lightcurves in a single energy band) make similar analysis impossible in the case of SPI-ACS. A detailed study of the SPI-ACS GRBs detected simultaneously with other satellites may help in recovering the intrinsic distributions of the GRB properties. Alternatively, selection biases might be assessed via a consideration of different trigger criteria. It has been shown that a sample of the short bursts may be better probed with the time-to-spill flux-based rather the traditional fluence-based trigger (Norris et al. 1984).

The second result of our work is the identification of a characteristic timescale of variability of long GRBs, see Fig. 5. This timescale can be roughly attributed to the typical separation between the individual pulses or activity periods of GRBs. It is interesting to note that the characteristic timescale of long GRBs coinsides with the timescale at which a break in the distribution of GRB durations is observed (see Fig. 3). This might indicate that many long GRBs are sequences of $\sim 1$ s pulses, with the overall burst duration determined by the overall number of pulses. The "pileup" in the distribution of GRB durations, at the timescale $\sim 20 \mathrm{~s}$ shows that long GRBs typically consist of $\sim 10-20$ pulses.

The majority of GRB with measured redshifts are situated at $z \sim 1-2$ (Salvaterra et al. 2012). This implies that the characteristic timescale in the GRB rest frame is of the order of $0.5 \mathrm{~s}$.

Our measurement of the characteristic pulse scale is robust but simplistic. Extensive studies of the individual pulse properties were performed by a number of groups (Norris et al. 1996; Hakkila \& Nemiroff 2009; Hakkila \& Preece 2011). In their pioneering work Norris et al. (1996), matched the observed lightcurves by a combination of model pulses. Notably, their result - the characteristic timescale of the order of a second, is close to the one we obtained with model-independed structure function analysis.

Results of Norris et al. (1996) and further studies (e.g. Hakkila et al. 2008; Ryde \& Svensson 2002) also indicated that the pulse properties (including characteristic timescales) are correlated. Since we use a wide range of the peak fluxes, it may should have an effect on our measurement of the characteristic one-second scale. However, this timescale is readily detectable in the SPI-ACS data even after smoothing introduced by ignoring the correlations. The robustness of the detection supports the idea that it should be associated with a real physical characteristic of the bursts.

The distinct characteristic timescale of the order of a second might be associated with the variability of the GRB central engine. In this case the remarkable persistency of the this timescale seems to be naturally attributed to the similarity of the progenitors (Beloborodov et al. 2000). Alternatively, it can be related to the size of the emission region. Further investigation of the nature of the characteristic timescale, exploiting the rich timing data of the SPI-ACS, is required to clarify its origin.

Acknowledgements. We would like to thank Nicolas Produit for the useful discussions of the subject and for the comments on the manuscript.

\section{References}

Abdo, A. A., Ackermann, M., Ajello, M., et al. 2009, ApJ, 706, L138

Adriani, O., Barbarino, G. C., Bazilevskaya, G. A., et al. 2011, Science, 332, 69 Aptekar, R. L., Frederiks, D. D., Golenetskii, S. V., et al. 1995, Space Sci. Rev., 71,265
Barthelmy, S. D., Chincarini, G., Burrows, D. N., et al. 2005, Nature, 438, 994 Battistoni, G., Cerutti, F., Fassò, A., et al. 2007, in Hadronic Shower Simulition Workshop, ed. M. A. R. Raja, AIP Conf. Ser., 896, 31

Beloborodov, A. M., Stern, B. E., \& Svensson, R. 2000, ApJ, 535, 158

Bhat, P. N., Fishman, G. J., Meegan, C. A., et al. 1992, Nature, 359, 217

Borgonovo, L. 2004, A\&A, 418, 487

Briggs, M. S., Paciesas, W. S., Pendleton, G. N., et al. 1996, ApJ, 459, 40

Cline, D. B., Matthey, C., \& Otwinowski, S. 1999, ApJ, 527, 827

Cline, D. B., Matthey, C., Otwinowski, S., Czerny, B., \& Janiuk, A. 2007, in The First GLAST Symposium, ed. S. Ritz, P. Michelson, \& C. A. Meegan, AIP Conf. Ser., 921, 280

Deluit, S., Produit, N., Mowlavi, N., Rohlfs, R., \& Dubath, P. 2004, in 5th INTEGRAL Workshop on the INTEGRAL Universe, ed. C. W. V. Schoenfelder, \& G. Lichti, ESA Spec. Publ., 552, 641

Fenimore, E. E., in 't Zand, J. J. M., Norris, J. P., Bonnell, J. T., \& Nemiroff, R. J. 1995, ApJ, 448

Ferrari, A., Sala, P. R., Fassò, A., \& Ranft, J. 2005, FLUKA: A multi-particle transport code, program version 2005 (Geneva: CERN)

Fynbo, J. P. U., Watson, D., Thöne, C. C., et al. 2006, Nature, 444, 1047

Gal-Yam, A., Fox, D., Price, P., et al. 2006, Nature, 444, 1053

Gehrels, N., Norris, J. P., Barthelmy, S. D., et al. 2006, Nature, 444, 1044

Hakkila, J., \& Nemiroff, R. J. 2009, ApJ, 705, 372

Hakkila, J., \& Preece, R. D. 2011, ApJ, 740, 104

Hakkila, J., Giblin, T. W., Norris, J. P., Fragile, P. C., \& Bonnell, J. T. 2008, ApJ, 677, L81

Heise, J., Zand, J. I., Kippen, R. M., \& Woods, P. M. 2001, in Gamma-ray Bursts in the Afterglow Era, ed. J. H. E. Costa, \& F. Frontera, 16

Hurley, K., Rau, A, von Kienlin, A., \& Lichti, G. 2004, in 5th INTEGRAL Workshop on the INTEGRAL Universe, ed. C. W. V. Schoenfelder, \& G. Lichti, ESA Spec. Publ., 552, 645

Hurley, K., Boggs, S. E., Smith, D. M., et al. 2005, Nature, 434, 1098

Hurley, K., Rowlinson, A., Bellm, E., et al. 2009, A new analysis of the short-duration, hard-spectrum GRB 051103, a possible extragalactic SGR giant flare

in 't Zand, J. J. M., \& Fenimore, E. E. 1996, ApJ, 464, 622

Klebesadel, R. W., Strong, I. B., \& Olson, R. A. 1973, ApJ, 182, L85

Kommers, J. M., Lewin, W. H. G., Kouveliotou, C., et al. 1998, unpublished [arXiv: astro-ph/9809300]

Kouveliotou, C., Meegan, C. A., Fishman, G. J., et al. 1993, ApJ, 413, L101

Lee, T. T., \& Petrosian, V. 1996, ApJ, 470, 479

MacFadyen, A. I., \& Woosley, S. E. 1999, ApJ, 524, 262

Mazets, E. P., Golenetskii, S. V., Ilinskii, V. N., et al. 1981, Ap\&SS, 80, 3

Mereghetti, S., Götz, D., Borkowski, J., Walter, R., \& Pedersen, H. 2003, A\&A, 411, L291

Mereghetti, S., Götz, D., Weidenspointner, G., et al. 2009, ApJ, 696, L74

Minaev, P. Y., Pozanenko, A. S., \& Loznikov, V. M. 2010, Extended Emission from Short Gamma-Ray Bursts Detected with SPI-ACS/INTEGRAL

Norris, J. P., \& Bonnell, J. T. 2006, ApJ, 643, 266

Norris, J. P., Cline, T. L., Desai, U. D., \& Teegarden, B. J. 1984, Nature, 308, 434

Norris, J. P., Nemiroff, R. J., Bonnell, J. T., et al. 1996, ApJ, 459, 393

Norris, J. P., Scargle, J. D., \& Bonnell, J. T. 2001, in Gamma-ray Bursts in the Afterglow Era, ed. J. H. E. Costa, \& F. Frontera, 40

Paciesas, W. S., Meegan, C. A., Pendleton, G. N., et al. 1999, ApJ, 122, 465

Parker, E. N. 1958, Phys. Rev., 110, 1445

Piro, L., Amati, L., Antonelli, L. A., et al. 1998, A\&A, 331, L41

Rau, A., Kienlin, A. V., Hurley, K., \& Lichti, G. G. 2005, A\&A, 438, 1175

Ryde, F., \& Svensson, R. 2002, ApJ, 566, 210

Sakamoto, T., Barthelmy, S. D., Baumgartner, W. H., et al. 2011, ApJS, 195, 2

Salvaterra, R., Campana, S., Vergani, S. D., et al. 2012, ApJ, 749, 68

Savchenko, V., Neronov, A., Beckmann, V., Produit, N., \& Walter, R. 2010, A\&A, 510, A77

Scargle, J. D. 1998, ApJ, 504, 405

Schneid, E. J., Bertsch, D. L., Fichtel, C. E., et al. 1992, A\&A, 255, L13

Segreto, A., Labanti, C., Bazzano, A., et al. 2003, A\&A, 411, L215

Shikaze, Y., Haino, S., Abe, K., et al. 2007, Astropart. Phys., 28, 154

Soderberg, A. M., Kulkarni, S. R., Nakar, E., et al. 2006, Nature, 442, 1014

Truscott, P. R., \& Dyer, C. S. 1997, in The Transparent Universe, ed. P. D. C. Winkler, \& T. J. L. Courvoisier, ESA Spec. Publ., 382, 339

Valle, M. D., Chincarini, G., Panagia, N., et al. 2006, Nature, 444, 1050

Vedrenne, G., Roques, J. P., Schönfelder, V., et al. 2003, A\&A, 411, L63

Viganò, D., \& Mereghetti, S. 2009 [arXiv: 0912 . 5329]

von Kienlin, A., Beckmann, V., Rau, A., et al. 2003, A\&A, 411, L299

Winkler, C., Courvoisier, T. J.-L., Di Cocco, G., et al. 2003, A\&A, 411, L1

Woosley, S. E., \& Bloom, J. S. 2006, ARA\&A, 44, 507

Zhang, B. 2011, C. R. Phys., 12, 206 